\title{
Estrategia Metacognitiva en el Aprendizaje Significativo Empleando los Cuentos Ilustrados
}

\section{Metacognitive Strategy in Significant Learning Using Illustrated Stories}

Ana Isabel García-Monroy ${ }^{1}$, Engelbert Eduardo Linares-González ${ }^{2}$ y Lucero Martínez-Allende ${ }^{3}$

\section{() \\ EDICIÓN: 进-CIVTAC}

Recibido: 22/julio/2020

Aceptado: 27/agosto/2020

Publicado: 25/septiembre/2020

我 México

${ }^{2}$ México

${ }^{3}$ México

\section{IIIIInstitución}

${ }^{1}$ Instituto Politécnico Nacional ${ }^{2}$ Instituto Politécnico Nacional ${ }^{3}$ Instituto Politécnico Nacional

\section{Correo Eletrónico}

'elinaresg@gmail.com

2agarciamo@ipn.mx

3lumartinez@ipn.mx

\section{(1) ORCID}

${ }^{1}$ https://orcid.org/0000-0002-2924-0118 ${ }^{2}$ https://orcid.org/0000-0003-4814-6562 ${ }^{3} \mathrm{https}$ //orcid.org/0000-0003-3646-0386

\section{Citar así: GAAPA / IEEE}

Linares-González, E., García-Monroy, A. \& Martínez-Allende, L. (2020). Estrategia Metacognitiva en el Aprendizaje Significativo Empleando los Cuentos Ilustrados. Revista Tecnológica-Educativa Docentes 2.0, 9(2), 231-239. https://doi.org/10.37843/rted.v9i2.168

E. Linares-González, A. García-Monroy y L. Martínez-Allende, "Estrategia Metacognitiva en el Aprendizaje Significativo Empleando los Cuentos Ilustrados", RTED, vol. 9, n. ${ }^{\circ}$, pp. 231238, sep. 2020.

\section{Resumen}

Ante las actuales exigencias sociales, los retos presentes en la educación exigen un enfoque humano a su vez de contar con contenidos de vanguardia, bajo este contexto, el docente, debe desarrollar e implementar diversas estrategias dinámicas orientadas a estimular procesos cognitivos, afectivos y psicológicos en el estudiante, por consiguiente, la enseñanza de estrategias metacognitivas emerge como una integración de prácticas dirigidas a fomentar un aprendizaje autorregulado y significativo, el presente trabajo planteó como objetivo proponer una estrategia metacognitiva encaminada a promover el aprendizaje significativo en alumnos de Educación Superior a partir de la elaboración de Cuentos Ilustrados. Para dar cumplimiento al objetivo planteado se propuso una metodología de carácter cualitativo y cuantitativo enfocado a conocer procesos metacognitivos empleados por un grupo conformado por 25 alumnos de educación superior quienes elaboraron un cuento ilustrado en el cual plasmaron conceptos teóricos y metodologías utilizadas en el análisis de instalaciones eléctricas industriales. La elaboración de cuentos ilustrados pone de manifiesto habilidades metacognitivas empleadas por los estudiantes al plasmarlos en historias a través de argumentos y elementos gráficos, este recurso no se contrapone con el trabajo formal y profundo dado que se fundamenta en conceptos previamente conocidos y estudiados. El propósito de la estrategia propuesta incide en el proceso de enseñanza aprendizaje, fomentando el desarrollo y ejecución de diversas estructuras mentales que activan conocimientos previos que se complementan con nueva información objeto de estudio.

Palabras clave: Aprendizaje significativo, cuento, estrategia, proceso metacognitivo.

\section{Abstract}

Given the current social demands, the challenges in education require a human approach in turn to have cutting-edge content, in this context, the teacher must develop and implement various dynamic strategies aimed at stimulating cognitive, affective and psychological processes in The student, therefore, the teaching of metacognitive strategies emerges as an integration of practices aimed at promoting self-regulated and meaningful learning, the present work proposed as an objective to propose a metacognitive strategy aimed at promoting meaningful learning in Higher Education students from the elaboration of Illustrated Tales. To comply with the proposed objective, a qualitative and quantitative methodology was proposed focused on knowing metacognitive processes used by a group made up of 25 higher education students who prepared an illustrated story in which they reflected theoretical concepts and methodologies used in the analysis of facilities. industrial electricals. The elaboration of illustrated stories reveals metacognitive skills used by students when translating them into stories through arguments and graphic elements, this resource is not opposed to formal and deep work since it is based on previously known and studied concepts. The purpose of the proposed strategy affects the teaching-learning process, promoting the development and execution of various mental structures that activate previous knowledge that is complemented with new information under study.

Keywords: Learning, creativity, illustrated story, didactic strategy, metacognitive process. 


\section{Introducción}

El docente en su trabajo e interacción con alumnos dentro del aula, pocas veces reconoce los procesos regulatorios implicados en el aprendizaje, existiendo un escaso conocimiento de las estrategias metacognitivas que permitan dar seguimiento a los procesos de aprendizaje que siguen los estudiantes durante su formación, por consiguiente, un desconocimiento en procedimientos y una escasa aplicación de estrategias metacognitivas impiden en la mayoría de los casos cumplir satisfactoriamente con los objetivos educativos que se buscan en el estudiante.

En el contexto educativo, la metacognición influye en el proceso de aprendizaje del estudiante quien tiene que ajustar sus procesos mentales para adquirir y utilizar los conocimientos que se presentan en el aula, este proceso se puede definir como una actividad consciente de introspección en el alumno en torno a cualquier aspecto cognitivo, se puede decir que es; pensar sobre pensar (Flavell, 1979).

La metacognición se relaciona con la capacidad de moderar el aprendizaje propio para lograr una mayor autoeficacia, incluye procesos de autorregulación, automonitoreo, autoevaluación y planificación, en otras palabras, es la capacidad que tienen las personas en planificar, seleccionar, analizar y utilizar estrategias para una situación determinada. Implica un análisis en la selección de información, un control y una evaluación, ya sea para afirmarla o modificarla de acuerdo con sus requerimientos contextuales (Klimenko \& Alvares, 2009).

El término "metacognición" es relativamente reciente, pero tiene precedentes importantes en figuras clásicas de psicología, como Lev Vygotsky, William James y Jean Piaget (Fox \& Riconscente, 2008). Es un acto de carácter multidimensional, de dominio general que se puede mejorar usando diversas estrategias instruccionales (Schraw, 1998). Tiene un componente reflexivo que hace referencia a lo que conoce la persona en torno a su propia cognición, y un componente de regulación, asociado al control del propio aprendizaje.

De acuerdo con lo que menciona Bruin (2012)., existen algunos rasgos característicos en personas que aprenden eficazmente, los cuales son: conocimiento sobre las limitaciones propias, la utilidad del ensayo y el aprendizaje distribuido, la capacidad de tener un amplio repertorio de estrategias y saber cómo y cuándo usarlas. El nivel de desarrollo de algunas de estas capacidades se encuentra asociadas con el coeficiente intelectual. Sin embargo, un aspecto relevante es dentro de una educación organizada, que contemple el uso de estrategias de aprendizaje efectivas, las cuales pueden compensar por mucho a las propias con las que cuentan las personas más inteligentes (Schraw, 1998).

Por otro lado, los procesos metacognitivos son de carácter interno, permiten en este caso, al estudiante definir un problema, planificar, monitorear, acceder a conocimiento previo haciendo una deserción entre lo relevante e ignorando lo irrelevante y evaluar su ejecución. Por ejemplo, como lo mencionan Palincsar y Brown (1984)., al momento de leer un texto, se emplean una serie de estrategias para mejorar la comprensión, las cuales incluyen conocer de antemano el propósito de la lectura, apropiarse del tema, activar el conocimiento previo relevante asociado al texto, prestarles atención a las ideas importantes, automonitorearse $y$ hacer inferencias.

Un ejemplo común dentro del aula, es la resolución de ejercicios matemáticos, en los cuales se parte de un planteamiento a un problema, posteriormente los alumnos tienen que realizar un proceso de análisis, para planificar la estrategia de solución y llevarla a la práctica, con esto asegurar una mayor probabilidad de éxito en la solución, en comparación en únicamente obtener una respuesta, siendo esto, una de las características en las metodologías de enseñanza tradicional que privilegia un aprendizaje superficial dejando a un lado un análisis más profundo.

De acuerdo con, lo mencionado por Muria (1994) se debe tener una mayor sensibilización en la relación enseñanza - aprendizaje, orientar a los estudiantes para que tengan una adecuada disposición a la construcción, desarrollo y afianzamiento de las competencias, reforzando su autoconfianza a partir de la motivación apropiada a la tarea o actividad que realizan. En este sentido, con el empleo de estrategias metacognitivas, se estimula de manera integral al estudiante, porque sacan mayor provecho al enfrentarse a problemas desconocidos, sin embargo, es importante proveer de manera simultánea las herramientas necesarias para que puedan resolverlos (Downing, Kwong, Chan, Lam \& Downing, 2008).

En este sentido, Rincón-Sierra (2020) para desarrollar la metacognición efectivamente es 
necesario que se estimulen las capacidades asociadas en el salón de clases, lo cual involucra un currículo basado en problemas lo que permitirá la construcción de un andamiaje apropiado que contemple no solamente estrategias metacognitivas al momento de estudiar, sino también inculcar la importancia de practicarlas consistentemente y en distintas áreas.

Aquellos profesores que reconocen la importancia de estimular un proceso reflexivo durante la enseñanza para maximizar el aprovechamiento estudiantil reconocen la aplicación pedagógica de este término (Joseph, 2003). Para lograr este desarrollo, existen una serie de estrategias de aprendizaje donde el estudiante es un sujeto activo, situado; y el docente, un mediador, entre el conocimiento y la construcción interna del mismo. Entre estas estrategias se encuentran: La redacción en la elaboración de escritos, el análisis de textos y elaboración de cuentos, los esquemas, mapas conceptuales, la elaboración de ideas, la formulación de preguntas, las propuestas de solución, el aprendizaje basado en problemas, y el desarrollo de competencias.

Todas estas estrategias favorecen el desarrollo de las características que de acuerdo con Mario Bunge (1964) son inherentes al pensamiento científico: objetivo, analítico, reflexivo, crítico, predictivo, trascendente, claro, preciso, simbólico, verificable y comunicable. El objetivo principal de esta investigación fue Proponer una estrategia metacognitiva que promueva el aprendizaje significativo en alumnos de educación superior a partir de la elaboración de cuentos ilustrados.

\section{Desarrollo}

La importancia de emplear estrategias metacognitivas en el salón de clases yace en su potencial para generar intervenciones pedagógicas que aumenten el grado de monitoreo y aprendizaje del estudiante, considerarlo como un aprendiz en busca de nuevos conocimientos, explorar nuevos contextos y afrontar situaciones en las que ponga en práctica sus conocimientos y habilidades, en este sentido, lograr que los alumnos aprendan a aprender y que lleguen a ser capaces de aprender de forma autónoma y autorregulada.

Esta visión en la enseñanza presenta un nicho de oportunidad y de interés al momento de planificar estrategias que permitan al estudiante desarrollar sus capacidades para aprender mediante la toma de conciencia y la adquisición de conocimiento, fortalecer habilidades y características particulares que facilitan su aprendizaje, las estrategias metacognitivas pueden emplearse con el fin de regular el proceso de aprendizaje.

En este sentido, la elaboración de cuentos ilustrados como estrategia metacognitiva, se puede considerar como una estrategia que favorece la motivación intrínseca de una manera amena $\mathrm{y}$ divertida, pues parte de la narración de una historia (contexto) a través de una sucesión de ilustraciones que se complementan con un texto escrito, el autor organiza la historia que quiere contar distribuyéndola en una serie de espacios, el objetivo es plasmar por medio del discurso o pensamiento de los personajes una trama que puede ser una contextualización de algún tema que se esté estudiando en clase, tomando como ingrediente principal la creatividad y participación del estudiante.

El empleo de cuentos ilustrados dentro del ámbito pedagógico favorece el aprendizaje a partir de una interacción entre el lector $\mathrm{y}$ el escritor, desarrollándose un proceso de comunicación, pues parte de la narración de una historia, en este sentido, como lo argumenta Velásquez-Ossa (2009) la naturaleza de la comunicación puede definirse desde el qué y el para que de la misma, se establece una acción y no como una cosa, en un sentido amplio, una acción es un proceso, por consiguiente, todo fin excluye su acción, por tanto acción $\mathrm{y}$ fin son diferentes.

Las estrategias o herramientas de enseñanzaaprendizaje, como lo argumenta Pimienta-Prieto (2012) son instrumentos de los que se vale el docente para contribuir a la implementación y el desarrollo de las competencias de los estudiantes, por lo tanto, la comunicación es una forma de interacción con el otro, entendiéndose al otro como a un semejente, en un sentido social, es algo más que informar, no es puro decir , sino un decir algo a alguien, un tener en común lo dicho, comunicar es dialogar, dar una información a alguien que la recibe y que la acepta como suya, se trata de un proceso interpersonal en el que se comparte algo (Yepes, 1996).

En este sentido, para Elisabeth K. Baur (1978) los medios de comunicación ilustrados como los cuentos o historietas son un medio de comunicación que tienden a presentar una mayor aceptación por 
parte de los adolescentes, por consiguiente $\mathrm{y}$ pedagógicamente se pueden ver como recursos que cuentan con diversas vertientes, con frecuencia, estos se asocian a elementos distractores por sus contenidos banales y sin importancia, que buscan entretener al lector, no aportando elementos valiosos, siendo esto un detractor al no ser consideradas como recursos pedagógicos.

Por otro lado, como lo argumenta Antonio Martín (1978) los cuentos ilustrados pueden verse desde un punto de vista positivo dentro de los recursos pedagógico, al estructurarse a partir de una historia narrada, complementada por dibujos y textos representados en una serie progresiva de momentos significativos según la selección temática hecha por el narrador. Esta característica apoya la incorporación de este medio de comunicación dentro de las estrategias metacognitivas, partiendo de elementos argumentados bajo un marco referenciado a un tema en específico y dirigido a reafirmar temas de clase.

Algunas de las características más relevantes que conforman los cuentos ilustrados son: La utilización apropiada de estructuras y elementos verbales para definir de manera adecuada la contextualización de la historia, una planificación acorde a hechos o situaciones a desarrollar a partir de los personajes dentro de la historia, acceder a conocimientos previos fomentando la capacidad de análisis del lector a través de la integración de conocimientos previos, monitorea el proceso de la comunicación tanto oral como escrita, favoreciendo la creatividad, estas características propician asimilar de una manera más significativa nuevos conocimientos.

\section{Metodología}

Para dar cumplimiento al objetivo planteado, se estableció una metodología de trabajo de tipo cualitativo y cuantitativo enfocado a conocer los procesos metacognitivos empleados por los estudiantes y el grado de aceptación de la estrategia, para esto, conto con la colaboración de un grupo de 25 alumnos de la carrera de ingeniería ambiental misma que se imparten dentro de la UPIBI, la estrategia se desarrolló dentro de la unidad de aprendizaje Ingeniería Electromecánica. Como lo marca el objetivo general de esta unidad, se espera que el alumno desarrolle un proyecto de iluminación en una planta de productos biológicos, realizando el análisis e interpretación de las instalaciones eléctricas industriales.

Al inicio del semestre se presenta la metodología de trabajo de la unidad de aprendizaje enfocada a promover los procesos metacognitivos a partir del trabajo colaborativo, se conformaron equipos de trabajo de máximo 5 alumnos. Con un avance del $70 \%$ en el programa de estudios se pide a los alumnos elaborar un cuento ilustrado, las instrucciones para su realización fueron la extensión de máximo de 10 páginas, dos viñetas mínimo por página, ilustraciones a color o blanco y negro, diálogos en globos, tomando como contexto inicial siguiente enunciado: "El héroe Electrón debe salvar a la galaxia UPIBIANA que cae en manos de las temibles cargas eléctricas. ¿Cómo salvara la galaxia el héroe Electrón?"

\section{Resultados}

Para conocer como el alumno llevó a cabo el proceso de regulación del conocimiento a partir de la elaboración de cuentos ilustrados y el uso de las estrategias de planeación, verificación y evaluación, se procedió a realizar una coevaluación a los trabajos desarrollados considerando los siguientes criterios:

1. Nivel de dificultad en la elaboración de la actividad.

2. Tipo y cantidad de información empleada.

3. Capacidad de selección y organización de la información empleada en el desarrollo de la historia.

Atendiendo el nivel de dificultad en la elaboración de la actividad desarrollada, el 95\% de los alumnos comentó que el trabajo desarrollado es creativo, permitiendo plasmar emociones $\mathrm{y}$ sentimientos a partir de las expresiones gráficas en los personajes de la historia, además de poner de manifiesto habilidades artísticas como lo son el dibujo, el uso del color y la expresión literaria, ver figura 1 . 


\section{Figura 1}

El héroe superelectrón en posición de ataque contra sobrecarga.

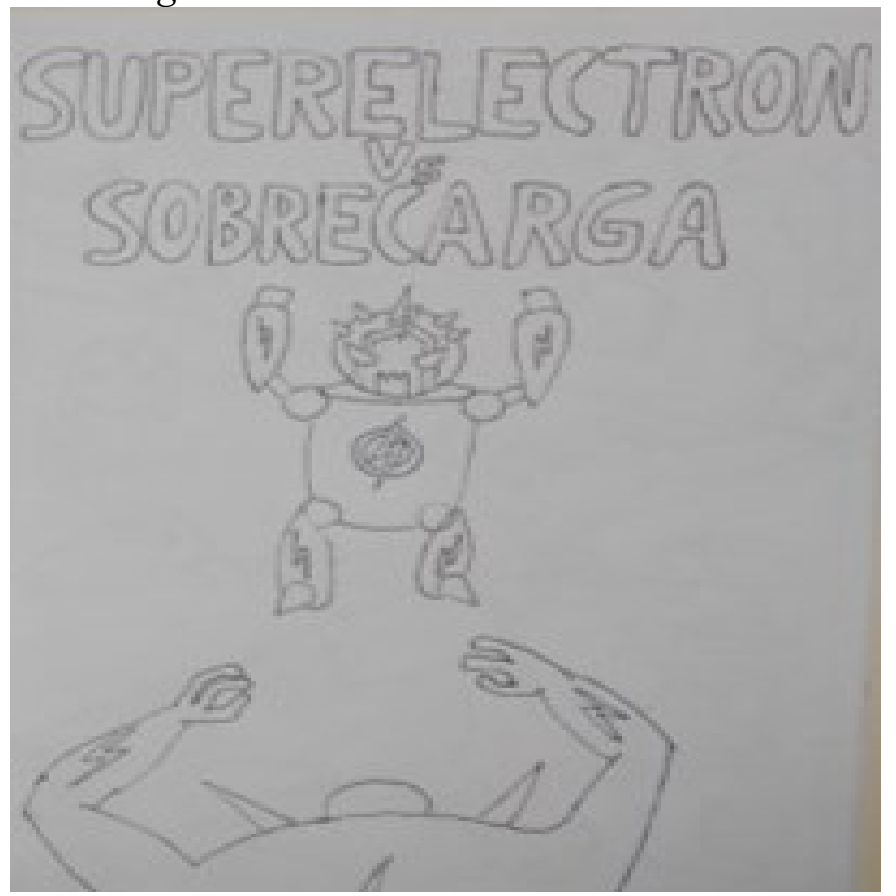

Nota. Manifiesto de habilidades artísticas, elaboración propia (2019).

Un 5\% de los alumnos que realizaron la actividad manifestó dificultad para expresar por medio de personajes en torno a una historia la conceptualización de elementos abstractos referentes a los temas de clase, por lo que se pronunciaron en realizar otro tipo de actividades tradicionales; como la ejecución de operaciones matemáticas, resolución de problemas, el repaso en libros de texto y tomar apuntes.

El 98\% de los alumnos expresó que la actividad representó un reto, por consiguiente, tuvieron que programar actividades, asignar tareas a cada integrante de equipo, llegar a acuerdos y resolver inconformidades en el interior de los equipos principalmente los relacionados con el desarrollo de las historias y la relación de conceptos de clase, por otro lado, la actividad hasta antes desconocida como recurso de estudio, apoyo en reforzar los temas de clase de manera amena, ver figura 2.

Un $2 \%$ de los alumnos, manifestó cierta indiferencia con la actividad desarrollada, esto plantea una pregunta en función de la estrategia propuesta. ¿Existe una relación con los estados emotivos o afectivos en el alumno y la actividad desarrollada? Una posible respuesta a este planteamiento se deriva de una posible asociación de

\section{Figura 2}

Desarrollo de la historia, relacionando temas de clase con la interacción de personajes.

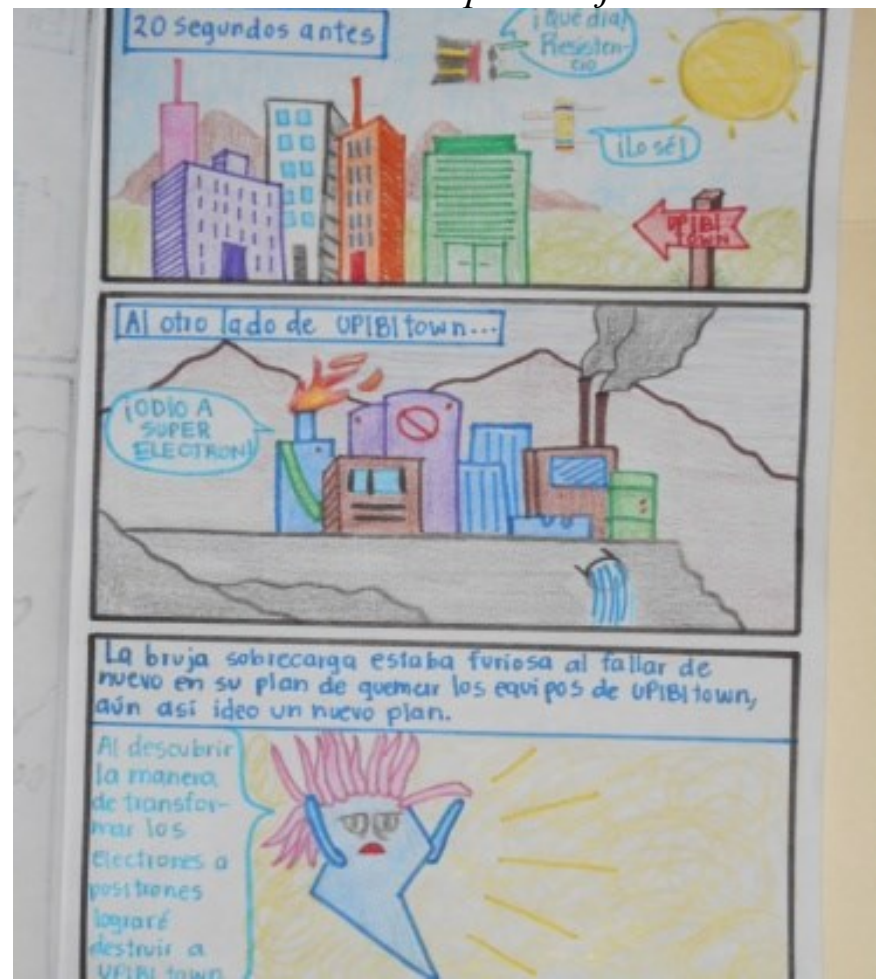

Nota. Apoyo en los temas de clase de manera amena, elaboración propia (2019).

diversos factores; como una motivación intrínseca de tal manera que, si el alumno presenta un desempeño pobre en una materia particular, será necesario averiguar qué elementos emotivos o motivacionales se relacionan con sus los hábitos de estudio.

Por otro lado, se encontró que el 100\% de los alumnos pone en práctica en mayor o menor grado los procesos metacognitivos, en este sentido el alumno reflexiona acerca de su propio pensamiento, analiza sobre si posee un tipo de conocimiento útil que sea fácilmente plasmado en el cuento, si está adquiriendo un nuevo saber o habilidad, reconoce el nivel cognoscitivo adquirido durante el proceso de elaboración del cuento, es consciente y autoevaluativo del nivel de conocimientos y habilidades adquiridos ver figura 3 . 


\section{Figura 3}

Manejo de emociones en los personajes plasmados en la trama del cuento.

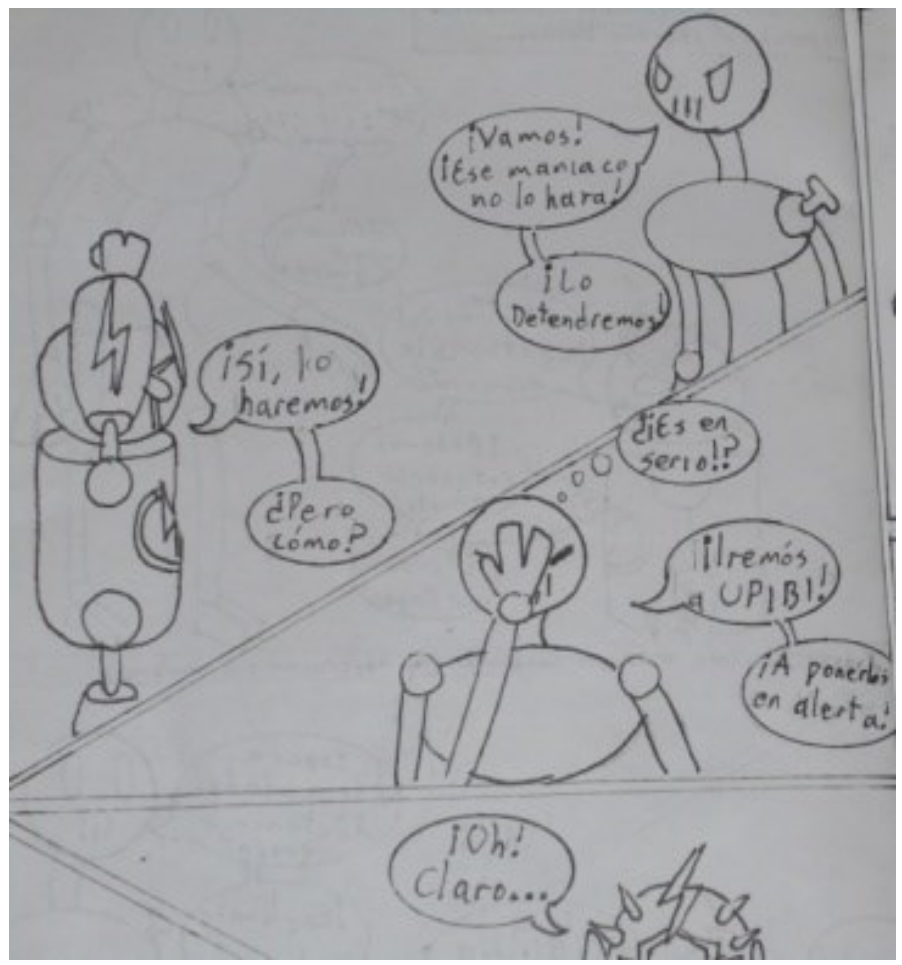

Nota. Nivel de conocimientos y habilidades adquiridas, elaboración propia (2019).

Finalmente, se les consultó en desarrollar una actividad semejante en otras unidades de aprendizaje, encontrándose lo siguiente:

1. El 90\% de los alumnos aseguro reproducir la actividad desarrollada en otras unidades de aprendizaje por la facilidad de recordar conceptos teóricos con los nombres de los personajes y las historias plasmadas.

2. El 85\% manifestó que existe una relación entre las historias y la secuencia de los temas que se presentan en el aula

3. Un 95\% manifestó que el desarrollo de la actividad represento todo un reto, por lo que no solamente pusieron en práctica conocimientos de la materia, también pusieron de manifiesto habilidades personales paro lograr el objetivo planteado, siendo esto algo que pocas veces se trabaja en otras unidades de aprendizaje.

4. Un $90 \%$ de los alumnos manifestó que esta actividad, permite aprende de una manera diferente y divertida pues todos trabajan y se apoyan en su elaboración.

5. El $98 \%$ de los alumnos expreso, "la actividad motivo nuestro lado creativo que muy pocas veces utilizamos durante nuestra estancia en la escuela, creemos que actividades como la desarrollada fortalece las habilidades blandas que requerimos al termino de nuestra carrera".

Para verificar que el objetivo planteado se alcanzó, es necesario evaluar el producto obtenido y corroborar el cumplimiento de cada una de las etapas del proceso metacognitivo, esto se observa a partir del análisis de la lista de cotejo mostrada en la tabla 1. Lista de cotejo de resultados de aplicación de la propuesta.

\section{Tabla 1}

Lista de cotejo de resultados de aplicación de la propuesta

\begin{tabular}{|c|c|}
\hline Aspecto para evaluar & Criterio \\
\hline $\begin{array}{l}\text { Los estudiantes emplean } \\
\text { fuentes adicionales de } \\
\text { información para la } \\
\text { elaboración de la actividad. }\end{array}$ & $\begin{array}{l}\text { Se consultan fuentes } \\
\text { adicionales a la } \\
\text { información trabajada en el } \\
\text { salón de clases. }\end{array}$ \\
\hline $\begin{array}{l}\text { Los estudiantes se } \\
\text { autoevalúan a fin de que } \\
\text { puedan reflexionar sobre } \\
\text { su propio aprendizaje. }\end{array}$ & $\begin{array}{lr}\text { Los } & \text { estudiantes } \\
\text { manifiestan destrezas más } \\
\text { allá de su desempeño } \\
\text { ordinario. }\end{array}$ \\
\hline $\begin{array}{l}\text { Los estudiantes emplean } \\
\text { estrategias basándose en la } \\
\text { reflexión cotidiana sobre } \\
\text { sus estrategias de estudio. }\end{array}$ & $\begin{array}{l}\text { La actividad se realiza a } \\
\text { partir del } \\
\text { colaborativo } \\
\text { momentos asignando } \\
\text { desarrollo. }\end{array}$ \\
\hline
\end{tabular}

Los estudiantes utilizan y La representación gráfica elaboran distintas formas de la información es de representación de la atractiva para los información en la actividad estudiantes.

desarrollada.

Los estudiantes adquieren Representan la información información en su memoria de forma secuencial a largo plazo mediante la presentando relación con asociación de conceptos, aquellos temas que ya palabras clave e imágenes.

Los estudiantes emplean Los estudiantes entienden estrategias que les que no se pretende que permitan recuperar memoricen información, información sobre un tema sino que se busque y que de manera organizada sean capaces de establecer estableciendo relaciones una relación clara con semánticas entre los aquello que han aprendido diferentes datos. con anterioridad.

Los estudiantes conocen $\mathbf{y}$ La realización de actividad practican habilidades de grupales permite socializar socialización y trabajo en temas que, de forma equipo. individual, resulta un tanto complejo abordarlos. 
Los estudiantes desarrollan habilidades necesarias para comprender y producir mensajes en distintas formas.

Nota. Análisis de la lista de cotejo, elaboración propia (2020).

\section{Discusión}

Al poner en práctica estrategias didácticas con un enfoque metacognitivo, se conduce a un aprendizaje significativo, por consiguiente, estas deben considerar al alumno como punto central del aprendizaje, deben promover la aplicación de recursos tanto cognitivos, afectivos y psicológicos, que pongan de manifiesto el comportamiento y desempeño del alumno dentro de una experiencia de aprendizaje

La elaboración de cuentos ilustrados refleja un proceso metacognitivo estructurado denotando el desarrollo de destrezas, habilidades y actitudes, establece una relación entre el conocimiento, lo que percibe el alumno y su entorno, facilita el aprendizaje de manera amena, abriendo vínculos de comunicación entre el alumno y el profesor. Este recurso no se contrapone con el trabajo formal y estricto de una investigación profunda pues tiene como base la utilización de saberes previamente adquiridos por el estudiante en combinación con nueva información. La elaboración adecuada de los cuentos ilustrados permite reforzar el aprendizaje, además de considerarse como un recurso didáctico de enseñanza aprendizaje innovador y que se puede adaptar a diversos temas.

Por otro lado, permite al docente poner en práctica recursos pedagógicos innovadores, dejando a un lado prejuicios, siendo una herramienta bastante útil en la enseñanza, el empleo de los cuentos ilustrados presenta una modalidad de lectura autónoma que invita al lector a interesarse en temas en los cuales los personajes se desenvuelven en un mundo de fantasía en el que habitan.

\section{Conclusiones}

Actualmente, el modelo educativo en el IPN a nivel superior hace poco énfasis en fomentar el desarrollo de estrategias de estudio eficaces en los alumnos, derivado de la cantidad de información la cual deben estructurar, sintetizar y asimilar, un porcentaje mínimo de esta tiene un carácter significativo en la formación del estudiante.
Por consiguiente, los alumnos deben implementar por cuenta propia estrategias de estudio de acuerdo con sus necesidades particulares y muchas veces estas no tienen la eficacia esperada, en el peor de los casos, los estudiantes que no desarrollan estrategias apropiadas demuestran un desempeño pobre y son más propensos a padecer afectaciones emocionales de frustración en el ambiente escolar.

Las estrategias didácticas con enfoque metacognitivo apoyan al docente a elaborar e implementar diversos recursos didácticos adecuándolos a las características y necesidades de los grupos en turno, estas estrategias facilitan la autorregulación de los procesos mentales de aprendizaje presentes en los alumnos.

El poner en práctica estrategias como la elaboración de cuentos ilustrados que toman como referencia postulados del metacognitismo, pueden considerarse como estrategias que incorporan técnicas, actividades, procedimientos e instrumentos que permiten motivar, activar y reforzar los conocimientos con respecto a la nueva información que va accediendo el alumno, siendo una tarea que no sólo implica el diseño de las actividades.

Pero se, involucra el seguimiento del proceso cognitivo del estudiante, el cual se puede autorregular a través de los procesos metacognitivos de planificar, controlar y evaluar, lo que permite optimizar el proceso de enseñanza y aprendizaje como el rendimiento académico, la motivación, y apropiación del conocimiento.

El empleo de los cuentos ilustrados, además de considerarse como un recurso de enseñanza aprendizaje de carácter innovador dada la integración de diversas habilidades y expresiones presentes, permite a los docentes explorar nuevos recursos a partir de elementos comunes y conocidos por los alumnos, dejando a un lado prejuicios negativos en su empleo, los cuentos ilustrados representan una modalidad de lectura de carácter autónomo que invita al lector a interesarse en los temas de una unidad de aprendizaje en particular a partir del desarrollo de la historia en los cuales los personajes se desenvuelven en el mundo de fantasía en el que habitan.

Finalmente, un punto de análisis que presenta la estrategia propuesta es la certidumbre en los resultados obtenidos, puesto que durante la elaboración y estructuración del cuento ilustrado están presentes factores tanto afectivos como emocionales presentes en el alumno y estos son plasmados en los trabajos, cabe la posibilidad de que si un estudiante presenta un bajo desempeño 
Estrategia Metacognitiva en el Aprendizaje

académico derivado a factores motivacionales, posiblemente sea le sea poco significativo la actividad por consiguiente el objetivo que se busca con la elaboración del cuento ilustrado no consiga el resultado esperado, por consiguiente, será necesario dar un acompañamiento transversal empleando otras estrategias en combinación con la estrategia propuesta.

\section{Referencias}

Baur, E. (1978) La historieta como experiencia didáctica, Ed. Nueva Imagen, México.

Bruin, A., \& Gog, T. (2012). Improving self-monitoring and selfregulation: From cognitive psychology to the classroom. Learning and Instruction, 22, 245-252.

Downing, K., Kwong, T., Chan, S., Lam, T., \& Downing, W. (2008). Problem-based learning and the development of metacognition.Higher Education, 57(5),609-621.

Flavell, J. (1979). Metacognition and cognitive monitoring: A new area of cognitive-developmental inquiry. American Psychologist, 34(10), 906-911.

Fox, E., \& Riconscente, M. (2008). Metacognition and self-regulation in James, Piaget, and Vygotsky.Educational Psychology Review, 20,373-389.

Joseph, N. (2003). Metacognition in the classroom: Examining theory and practice. Pedagogy: Critical Approaches to Teaching Literature, Language, Composition, and Culture, 3(1), 109114.

Klimenko, O. \& Alvares, J. L. (2009). Aprender cómo aprendo: la enseñanza de estrategias metacognitivas. Colombia: La Sabana Ediciones.

Martin A. (1978) Historia del cómic español: 1875-1939, Ed. Gustavo Gil, Barcelona.

Merton, R. K., Torner, F. M., Borqués, R., \& Bunge, M. (1964). Teoría y estructura sociales (No. 04; HM101, M4.). México: Fondo de Cultura Económica.

Muria, I. (1994). La enseñanza de las estrategias de aprendizaje y las habilidades

metacognitivas. México:Perfiles Ediciones.

Palincsar, A., \& Brown, A. (1984). Reciprocal teaching of comprehension-fostering and comprehension-monitoring activities. Cognition and Instruction, 1(2), 117-175.

Rincón-Sierra, F. M. (2020). Análisis de la aplicación de la teoría cognitiva de Jerome Bruner como mecanismo para fortalecer la conducta ambiental en los estudiantes del Grado Segundo de la Institución. Revista Tecnológica-Educativa Docentes 2.0, 9(1), 132-141. https://doi.org/10.37843/rted.v9i1.110

Schraw, G. (1998). Promoting general metacognitive awareness. Instructional Science, 26, 113-125.
Pimienta-Prieto, J. (2012). Estrategias de enseñanza-aprendizaje Docencia universitaria basada en competencias, Ed. Pearson educación. México.

Tobón, S. (2006). Docencia Metacognitiva y Competencias del Profesorado. Barcelona: Tributo Ediciones.

Velásquez-Ossa, C., López Jiménez, D., \& Gómez Giraldo, J. (2009). La naturaleza de la comunicación: un aporte a su discusión conceptual.

Yepes, Ricardo.1996. Qué es eso de la filosofía. De Platón a hoy. Barcelona: Ediciones del Drac. 